\title{
Analysis and Alternate Selection of Nanopowder Modifiers to Improve a Special Protective Coating System
}

\author{
S. P. Bardakhanov, ${ }^{1,2,3}$ V. N. Goverdovskiy, ${ }^{3,4}$ C.-M. Lee, ${ }^{4}$ O. C. Lee, ${ }^{4}$ and V. T. Lygdenov ${ }^{2,5}$ \\ ${ }^{1}$ Aerophysics of Subsonic Flows Laboratory, Institute of Theoretical and Applied Mechanics, Russian Academy of Science, \\ 4/1 Institute Str., Novosibirsk 630090, Russia \\ ${ }^{2}$ Advanced Materials Laboratory, Buryat State University, 24a Smolyn Str., Ulan-Ude 670000, Russia \\ ${ }^{3}$ Engineering Ecology Department, Novosibirsk State Technical University, 20 K. Marx Ave., Novosibirsk 630073, Russia \\ ${ }^{4}$ School of Mechanical and Aerospace Engineering, University of Ulsan, 29 Mugue-Dong, Nam-Gu, Ulsan 680749, Republic of Korea \\ ${ }^{5}$ Institute of Physics of Materials Science, 6 Sakhyanov Str., Ulan-Ude 670047, Russia
}

Correspondence should be addressed to S. P. Bardakhanov; bard@itam.nsc.ru and V. N. Goverdovskiy; vng_scien@yahoo.com

Received 15 November 2016; Revised 5 March 2017; Accepted 16 March 2017; Published 16 May 2017

Academic Editor: Brian Wigdahl

Copyright ( 2017 S. P. Bardakhanov et al. This is an open access article distributed under the Creative Commons Attribution License, which permits unrestricted use, distribution, and reproduction in any medium, provided the original work is properly cited.

This paper presents a practical approach for rational choice of silica nanopowders as modifiers to control and improve the performance of protective coating systems operating in harsh environmental conditions. The approach is based on the multiparameter analysis of nanoparticle reactivity of similar silica synthesized by using chemical and physical methods. The analysis indicates distinct adsorption centers due to the differences in the particles formation; the features of the formation and adsorption mechanisms lead to higher diffusion capacity of the nanoparticles, synthesized by physical methods, into a paint material and finally result in stronger chemical bonds between the system elements. The approach allows reducing the consumption of paint materials by $30 \%$ or more, at least $2-3$ times increasing of the coating adhesion and hence the system life. Validity of the approach is illustrated through the data obtained from comparative modeling, factory testing, and practical use of modified systems.

\section{Introduction}

Transport vehicles and infrastructural facilities and other machines and structures operating in harsh environmental conditions need to be protected against mechanical, thermal, and seawater impacts. Despite substantial progress, the protective coating systems (PCSs) made by using off-the-shelf alkyd, epoxy, and other types of single- or multicomponent primers and paints do not withstand long-term impacts. Therefore, the operational characteristics of such PCSs can fail long before the service life predicated by industrial standards. Repeated repairing or new paint application is labor intensive, costly, and environmentally harmful. Therefore, these works are impracticable, in many cases.

Modification of a paint material by using different nanopowders is an effective approach for improvement of a PCS property (see, e.g., [1]). The modifiers are used as antisettling agents to extend storage and application time of paint materials; they can increase the substrate and interlayer adhesion and cohesion, prevent corrosion, provide color retention, and maintain many other properties with a long life time. For instance, zinc nanopowders are used as the anticorrosive agents [2], the heat insulation effect of a paint coating can be improved by adding the iron oxide nanopowders [3], and nanotitanium oxides are used for selfcleaning coatings [4]. However, silica nanopowders are most often used among the modifiers [5-7]. Different synthetic amorphous silica and relevant nanotechnologies can provide improvement of the operational characteristics of PCSs in a wide range [8-18].

There are many methods to produce silica nanopowders that are all-important for industries. These amorphous 
nanopowders are produced by chemical and physical methods. The chemical silica nanopowder (CSNP) can be synthesized, for example, from silicon tetrachloride (chlorosilane) by the hydrolysis of its vapor phase in a hydrogen-oxygen flame. The hydrolysis variables determine the properties of the CSNP, such as porosity, specific surface area, particle size, and shape $[19,20]$. Among the physical methods, high-temperature and evaporation of silica ingot are highly effective and have proved to be promising for practical applications. By using the electron beam, such physical silica nanopowder (PSNP) is synthesized by the vaporization at a certain temperature, followed by cooling of the vapor, condensation in air, activation, and finally separate collection of the micro- and nanoparticles in a range. The particle parameters are regulated by the relationship between the power and gas flow parameters [21, 22].

However, silica nanopowders with analogous or similar general characteristics have significantly different chemical activity that leads to different levels of efficiency as the PCS modifiers. Underestimation of these features can result in lower performance of modified PCSs even in comparison with conventional (unmodified) PCSs in the same operating conditions. Besides, this leads to complication of the requirements to selection and utilization of the modifiers that are reasonable in terms of usefulness and cost during the paint application.

This paper presents a practical approach for rational choice of silica nanopowders to control and improve the performance of a PCS operating in harsh environmental conditions for a long time. The approach is based on the data of systematical multiparameter comparative analysis of similar silica nanopowders. This resulted in certain relations between the features of the particles formation by using chemical and physical methods and reactivity of the particles. Essential differences are indicated in the adsorption mechanisms, the energy spectra, and diffusion capacity of the surface associated layers of the CSNP and PSNP particles. Then, comparative levels of adhesion and cohesion are estimated between the elements of the PCS reference specimens for different paint compositions with CSNPs and PSNPs. Further, the factory paint application and comprehensive test of the coatings, modified with the CSNP or PSNP, are carried out to evaluate the levels of technical efficiency and practical value of CSNPs and PSNPs use. Practical payoffs of the approach application are demonstrated through the results obtained from a long-time operation of modified PCSs in harsh and extreme environment.

\section{Experimental}

2.1. Materials. Off-the-shelf paint materials, in particular, the primers EP1760 and EP170, and topcoat UT6581 were utilized in modeling studies and factory testing. These are two-component, polyamide cured epoxy resin based primers containing zinc phosphate as corrosion inhibiting pigment and quick drying type polyurethane resin based finish coat, respectively [23]. In modeling studies, the epoxy resins DER 330 and ED-20 (hereinafter E1 and E2) [24, 25] and the distillated water were utilized as well. Commercial silica nanopowders Aerosil ${ }^{\circledR}$, which have specific surface area of $S_{\mathrm{CSNP}}=\{90,120,200,300,380\} \mathrm{m}^{2} / \mathrm{g}$, and Tarkosil ${ }^{\circledR}, S_{\mathrm{PSNP}}=$ $\{50,80,100,120,150\} \mathrm{m}^{2} / \mathrm{g}$, respectively, were used $[20,21]$. When using the measured densities, the volume fractions of these nanoparticles were calculated from the known weight percentages. Then, the nanopowders were supplied and premixed with either base A of the paint materials (in factory testing) or curing agent B of the resins (in modeling studies) using a vibrating-pulsed mechanical stirrer. As expected, the viscosity of the mixtures increased differently when using the nanopowders. Therefore, the viscosity values of the mixtures were equalized by adding either base A or curing agent B, respectively.

2.2. Material and Coating Characterization. In modeling studies, the specific surface areas and average particle sizes for the nanopowders were measured by liquid nitrogen absorption method (BET-analysis) when using standard procedure. $\mathrm{X}$-ray emission spectroscopy was employed to measure the energy $\operatorname{SiK} \alpha$ and $\mathrm{OK} \alpha$ spectra of the nanoparticles and thus to compare the forces of chemical bonds in the modified liquid compositions [26-28]. The potentials were measured in reverse AOT micelles (aqueous dispersions) for silica nanoparticles $[29,30]$. To avoid the particle agglomeration, each nanopowder sample was exposed with ultrasound for $30 \mathrm{~min}$, after that it was dispersed. Viscosity measurement of a mixture was held at a certain concentration of a nanopowder, and the relative viscosity was calculated by dividing the measured data to the viscosity of a pure (initial) liquid at the same temperature [31]. The nanoparticle surface reactivity was studied using the adsorption of acid-base indicators with different inherent $\mathrm{pK}_{a}$-values in the range of -5 to +15 , and then assays with corresponding $\mathrm{p} K_{a}$-values were selected [32]. The method is based on spectrophotometric measurement of optical densities for standard aqueous solutions of the indicators at wavelengths corresponding to their light absorption maxima. Transmission electron microscopy (TEM), together with software for the instrument, was used for measurement of particle size distribution. Atomic force microscopy (AFM) was employed to study the strength of a coating when using a primer or paint and the same paint materials premixed with the nanopowders [14].

In factory testing, the test program included instrumental measurement, a comprehensive comparison and control of the PCS technological and operational characteristics, such as the wet film thickness (WFT) and dry film thickness (DFT), adhesion, and corrosion resistance to salt water. Each test sample included at least 30-50 replicates per material type. Patented procedures and tools for production of PSNPs and liquid compositions were used as well $[33,34]$. The WFT and DFT values were measured according to the standard methods $[35,36]$. The adhesion control was carried out by the cross-cut and pull-off methods which are most often used in practice [37, 38]. However, these methods are not accurate enough and unrepresentative especially at initial period of a PCS operation. Therefore, we have added the study with 
the micrographic test [39]. This technology provides a high accuracy of comparative analysis and allows predicting and control performance deterioration of a PCS. An accelerated test was done to study the PCS resistance to salt water. The test included an assessment of the density of air-cells and airwater bubbles that arisen under a paint coating and also the breadth of the corrosion spread under the coating when using the cross-cut method [40]. A periodic inspection of operating PCSs to control and prevent degradation of their operational characteristics (adhesion strength, coating thickness, appearance, ability to self-cleaning, etc.) was carried out as well.

\section{Results and Discussion}

The hypotheses and theoretical models were approved through the laboratory modeling studies and factory testing of the PCS specimens (structural or stainless steel plates coated properly) and continuous operation of the full-scale PCSs for the transport vehicles. The experiments were carried out through comparative testing of the paint materials and proper coatings modified with different types of CSNPs and PSNPs. The specimens and PCSs have been made and studied at identical test conditions including indoor and outdoor parameters.

3.1. Model Studies. The studies showed that the surface structures of CSNPs and PSNPs are quite different. These features can result in different reactivity with other conditions being equal. For instance, X-ray analysis showed that the CSNPs and PSNPs are amorphous. However, the PSNPs have less crystallinity than the CSNPs. Further, the phase analysis showed that the PSNPs contain two phases of shortrange order, including the dominant $\mathrm{SiO}_{2}$ (96-901-349, spatial group PI Triclinic) and $\beta-\mathrm{SiO}_{2}$ (96-951-0148, spatial group PI Triclinic) [26]. Then, the structure of these two phases, sizes of the crystallinity domains, and atomic composition inside the domains were investigated by the Rietveld method [27]. The differences in the quantitative ratio of the phases gave an idea of why the PSNPs have higher specific reactivity than the CSNPs. This also raises the question of how significant the features of CSNPs and PSNPs and how these can be used to control the structural constraints and chemical bonds of a coating to improve the operational characteristics of a PCS.

3.1.1. Differences in the Surface Reactivity of Nanoparticles. Spectrophotometric measurements of the optical densities of solutions indicated the presence of adsorption centers of different types on the particle surfaces, including Lewis centers (the constant value is $\mathrm{p} K_{a}<0$ ), formed by oxygen atoms, and acids ( $\mathrm{p} K_{a}>14$ ), formed by silicon atoms, as well as Brønsted acid ( $\left.\mathrm{p} K_{a} \sim 0-6\right)$, neutral ( $\left.\mathrm{p} K_{a} \sim 6-8\right)$, and Lewis centers corresponding to hydroxyl groups. Figure 1 illustrates the features of adsorption centers of the CSNP and PSNP particles with close values of specific surface area $\left(S_{\mathrm{CSNP}}=\right.$ $90 \mathrm{~m}^{2} / \mathrm{g}$ and $S_{\mathrm{PSNP}}=100 \mathrm{~m}^{2} / \mathrm{g}$ ).

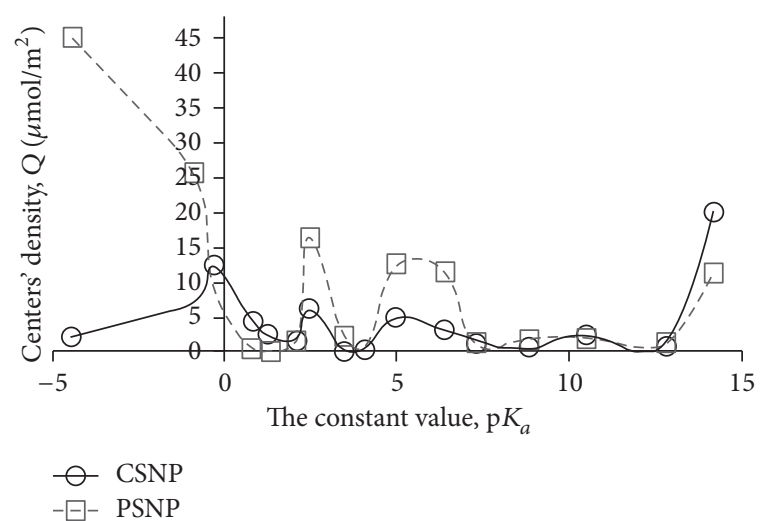

FIGURE 1: Distribution of adsorption centers on the nanoparticle surfaces.

The CSNP particle surface is mainly occupied by Lewis centers $\left(\mathrm{p} K_{a} \sim 14.2\right.$, silicon atoms or oxygen vacancies), relatively weak Lewis centers $\left(\mathrm{p} K_{a} \sim-0.3\right.$, probably corresponding to oxygen atoms in siloxane groups), and strong Brønsted acidic groups ( $\mathrm{p}_{a} \sim 0-2$, hydroxyls bound with electron-accepting Si atoms with high Lewis acidity). In contrast, the PSNP particle surface contains more strong Lewis centers $\left(\mathrm{p} K_{a} \sim-4.5\right.$ to -0.9 , oxygen atoms or silicon vacancies), Brønsted acidic groups $\left(\mathrm{p} K_{a} \sim 2.5-6.5\right)$, and weakly basic hydroxyl groups $\left(\mathrm{p} K_{a} \sim 8.8\right)$. The properties of the PSNP are determined by oxidation in the electron beam evaporation, and evidently these features are affected by the mechanisms of the particle formation, temperature range, and radiation [32].

The energy spectra of the CSNP and PSNP particles were measured by X-ray emission spectroscopy. The $\mathrm{SiK} \alpha$ and $\mathrm{OK} \alpha$ allowed comparing the forces of chemical bonds. The PSNP SiK $\alpha$ spectrum shifted by $0.1-0.3 \mathrm{eV}$ into the shortwave region in comparison with the same region of the CSNP $\mathrm{SiK} \alpha$ spectrum; besides, the PSNP region is wider. The energy level shifts, $\Delta \mathrm{Si} 2 \mathrm{p}$ and $\Delta \mathrm{Ols}$, of the skeleton electrons and the shifts, $\triangle \mathrm{SiKLL}$, in the Auger spectra were measured and compared (see Figure 2).

This analysis showed that the shifts in the PSNP's spectra reach $1 \mathrm{eV}$. In addition, the shifts of CSNP and PSNP have both positive and negative polarities relative to the spectra of natural quartz. The PSNP with specific surface area of $S_{\mathrm{PSNP}}<$ $100 \mathrm{~m}^{2} / \mathrm{g}$ reveals the binding energy, which is comparable to the extreme energy value for the CSNP with the highest specific surface area. For instance, the PSNP with specific surface area of $S_{\mathrm{PSNP}}<100 \mathrm{~m}^{2} / \mathrm{g}$ has the energy level shifts of $\Delta \mathrm{Si} 2 \mathrm{p}=0.6, \Delta \mathrm{Ols}=0.5$, and $\Delta \mathrm{SiKLL}=-0.8$, while these shifts for CSNP of $S_{\mathrm{CSNP}}=380 \mathrm{~m}^{2} / \mathrm{g}$ are $0.9,1.0$, and -1.3 , respectively [28]. Thus, the PSNP particles have more absorption centers on the silicon atoms' surfaces and on the oxygen atoms than the CSNP particles. The CSNP reveals a selective surface absorption of oxygen atoms, while the PSNP absorption capacity is based on weaker monovalent bonds. This difference is confirmed with measurement of $\xi$-potential values. The potentials were measured in reverse AOT micelles 


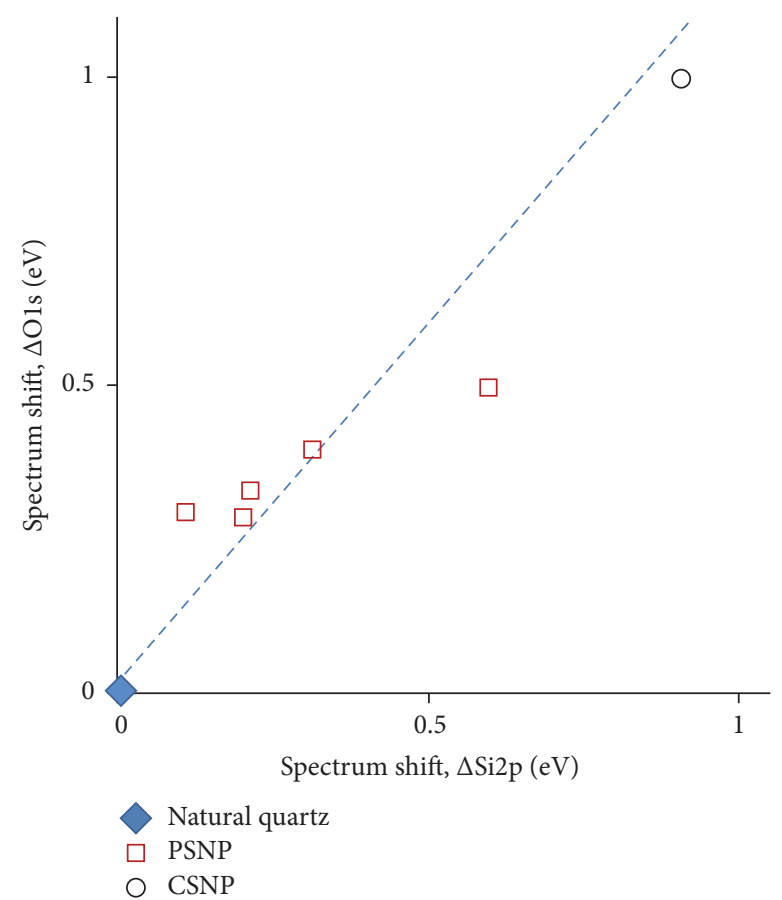

(a)

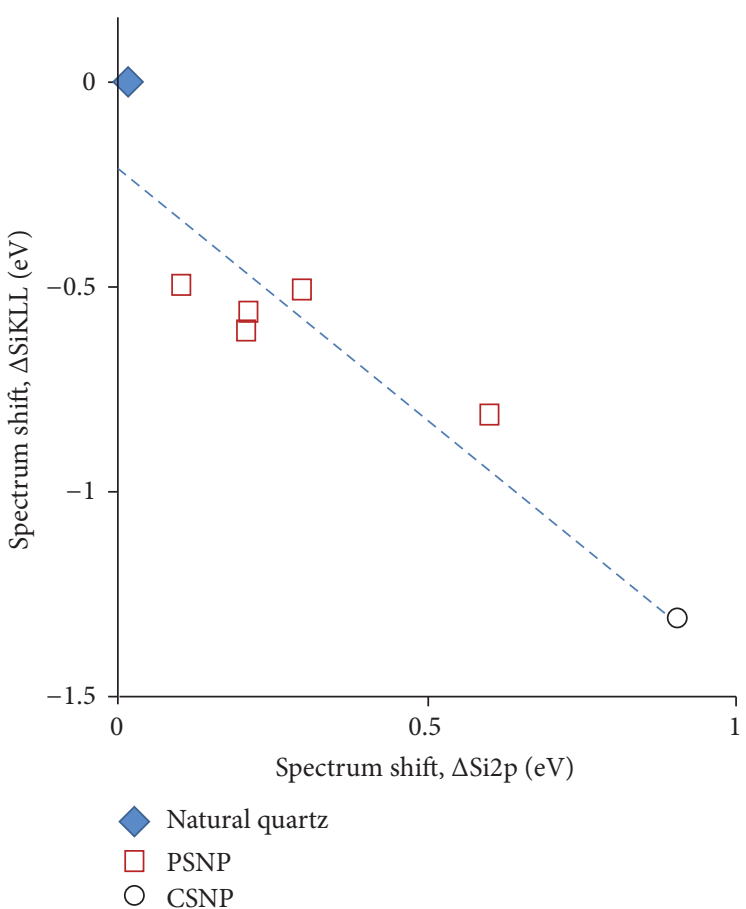

(b)

Figure 2: X-ray emission spectroscopy of natural quartz, PSNP, and CSNP, where there are (a) shifts of the O1s and Si2p spectra and (b) shifts of the Auger and Si2p spectra.

(aqueous dispersions) as $-73 \mathrm{mV}$ for PSNP and $-27 \mathrm{mV}$ for CSNP $[29,30]$. In the PSNP, the surface quadrivalent ions increase the electrostatic attraction couplings, which cause greater compression of the diffusion layer and reduce $\xi$ potential. This results in stronger chemical bonds between the dispersion medium and solid phase.

3.1.2. Associated Layer and Its Effect on the Particle Reactivity. The size and concentration of nanopowder change thixotropy and viscosity of a paint composition and can differently affect the coting film forming. This can be explained by a simple model of the surface reactivity of a nanoparticle in vicinity of its so-called "associated layer."

The relative viscosity $\mu / \mu_{0}$ of a liquid composition can be estimated by the Batchelor model [41]:

$$
\frac{\mu}{\mu_{0}}=1+2.5 \psi_{p}+6.25 \psi_{p}^{2}
$$

where $\mu_{0}$ and $\mu$ are the viscosities of an initial liquid and the liquid composition with a dispersed-phase and $\psi_{p}$ is the dispersed-phase volume concentration.

However, the experimental study and practical application of different nanopowders as the modifiers show that $\mu / \mu_{0}$ is much higher than a value calculated by (1). The reason is the associated layer of a thickness $\delta$ that is formed on the particle surface due to inner cohesive chemical bonds.
The effective bulk concentration of associated layers for $N$ particles in such a liquid composition can be assessed as

$$
\begin{aligned}
& \psi_{\mathrm{A}-\mathrm{L}}=\frac{V_{p} \cdot N}{V_{\Sigma}}=\frac{\pi \cdot N}{6 V_{\Sigma}} \int_{0}^{+\infty}(D+2 \delta)^{3} f(D) \mathrm{d} D \\
& \approx \psi_{p}\left[1+6 \delta \frac{E_{f(D)}\left(D^{2}\right)}{E_{f(D)}\left(D^{3}\right)}+\cdots+12 \delta^{2} \frac{E_{f(D)}\left(D^{2}\right)}{E_{f(D)}\left(D^{3}\right)}\right. \\
&\left.+8 \delta^{3} \frac{1}{E_{f(D)}\left(D^{3}\right)}\right] \approx \psi_{p}(1+K),
\end{aligned}
$$

where $V_{p}$ and $V_{\Sigma}$ are the volumes of the associated layers and the composition, respectively; $D$ is the average size of the particles; and $E_{f(D)}\left(D^{X}\right)=\int_{0}^{+\infty} D^{X} f(D) \mathrm{d} D$ is the $X$ th initial moment of the particle size distribution $f(D)$.

From the second term in the brackets of (2), the specific surface area can be determined as

$$
S_{\mathrm{sp}}=\frac{6 E_{f(D)}\left(D^{2}\right)}{\rho_{p} E_{f(D)}\left(D^{3}\right)},
$$

where $\rho_{p}$ is the particle density.

Substituting (2) into (1) yields more accurate value of the relative viscosity [42]:

$$
\frac{\mu}{\mu_{0}} \approx 1+2.5 \psi_{p}(1+K)+6.25 \psi_{p}^{2}(1+K)^{2} .
$$




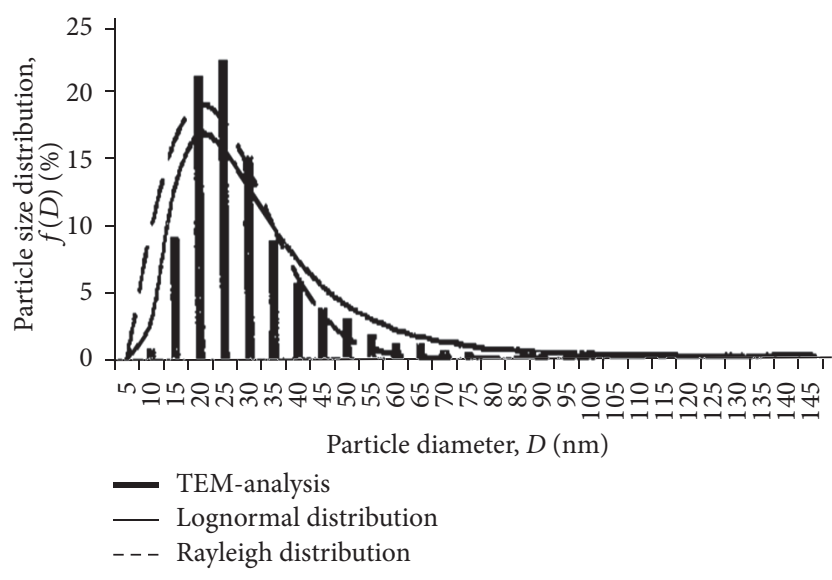

FIGURE 3: Particle size distribution for the PSNP obtained from the TEM data analysis.

A particular value of the correction factor $K$ can be obtained from analysis and approximation of experimental data in modeling the associated layers. For instance (see Figure 3), one can apply the Rayleigh function as a close approximation when using the formulation of $f(D)$ as the particle size distribution, which can be obtained from the TEM-analysis. Finally, the following approximation can be as follows:

$$
K \approx \delta \rho_{p} S_{\mathrm{sp}}+\frac{\pi}{8}\left(\delta \rho_{p} S_{\mathrm{sp}}\right)^{2}+\frac{\pi}{48}\left(\delta \rho_{p} S_{\mathrm{sp}}\right)^{3},
$$

when using $f(D)=D / \sigma^{-2} \exp \left(-0.5 D^{2} \sigma^{-2}\right)$ and the estimation of (3) as $S_{p}=\sqrt{8 \pi^{-1}}\left(\sigma \rho_{p}\right)^{-1}$, where $\sigma$ is the deviation. The product $\left(\delta \rho_{p} S_{\mathrm{sp}}\right)^{Y}$, where the index is $Y=1,2,3, \ldots$, means that an increase in concentration and decrease in the size of nanoparticles can drastically increase the viscosity of a liquid composition.

Thus, (4)-(5) could be considered as a universal model to estimate the viscosity of a liquid composition despite the size of the solid phase. If $K \neq 0$, then we deal with nanoparticles or their agglomerates; $K=0$ concerns microparticles which are dispersed in a liquid medium.

3.1.3. Comparative Diffusion Capacity of Associated Layers. The diffusion capacity of CSNP and PSNP depends on the properties of their particle surfaces and the activity of the surface functional groups as illustrated with various samples [31]. Figure 4(a) presents the experimental data to obtain relations between the size of the CSNP and PSNP particles and the thickness of associated layers in various liquid media. The thickness values are close to the results of (4)-(5). For instance, these are 6.37 and $7.1 \pm 0.8 \mathrm{~nm}$ for the PSNP when using the epoxy resins, E1 and E2, and $6 \pm 1 \mathrm{~nm}$ in the water. However, there is an obvious dependence of the layer thickness and the particle size for the CSNP. Figure 4(b) shows that (5) is suitable for analysis of the liquids containing such modifiers. If the particle diameter is less than the layer thickness, then the particle is not being capable of holding it. For this reason, the layer thickness is also reduced [42].
Figure 4(b) also shows how the viscosity depends on the nanopowder concentration. These results take into account the $\mathrm{K}$-factor correction of the increasing weight fraction of the particles due to the associated layer. Selection of the Kfactor calculated for each type of the liquid compositions meets the factor values obtained from the experiment. These differences of CSNP and PSNP result in much higher viscosity for liquids with the CSNP. This occurs in the range of $m_{\mathrm{np}}=$ $0-11$ wt.- $\%$ of nanoparticles.

The analysis showed that the reactivity of the CSNP and PSNP, which have distinct adsorption centers, with a dispersive liquid is quite different. In the above examples, the liquid materials thicken more, and the viscosity of the composition increases faster with increasing the CSNP concentration. In practice, this can take more time and power inputs to make well-mixed dispersion, as well as more refined dosing of thinner. However, the polymer film solidification of a paint coating modified with the PSNP can occur faster and with smaller loss of volatile components due to the faster formation of the chemical bonds.

3.1.4. Control and of the Strength of Structural Constraints and Chemical Bonds in a PCS. Adhesion and cohesion are key parameters of a PCS; they are determined by the PCS structure and by strength of structural constraints and chemical bonds between the PCS elements. Generally, numerous mechanical and thermal microdefects on a painted surface are responsible for interaction between a coating film and the surface. A model in Figure 5(a) illustrates that such PCS contains a certain number of microelements 4 (fillers, color pigments, etc.) on a painted surface 1 . If the microelements are smaller than the surface microdefects 2 , then the film 3 holds them. Otherwise, the coating film degrades fractally during operation of the PCS and loses a part of these microelements that results, for example, in thinning and color fading. The nanomodifiers increase the strength of structural constraints and chemical bonds. First, the solid nanoparticles and their agglomerates 5 (Figure 5(a)) form a number of redundant constraints between the PCS elements. These redundant constrains (like pins) slow down degradation of the PCS operational performance. Second, the modifiers increase the strength of chemical bonds and thus the cohesion between the coating layers.

The AFM showed the differences between the coatings before and after the modification. Figure 5(b) shows that the unmodified coating has defects with size of about $2.5 \mu \mathrm{m}$. The PSNP (Figure 5(c)) provided the synthesis of redundant structural constrains and chemical bonds that reduced the microdefects, which became smaller than $1 \mu \mathrm{m}$ [14].

3.2. Factory Comparative Experiments. The paint application and comparative testing of the coatings, modified with the CSNP or PSNP, showed how much the above hypotheses and models are valid. In addition, the consumption and spreading rate of the paint materials with and without modifiers were analyzed in comparison. The polyurethane topcoat (hereinafter TC) and polyamide epoxy primers (hereinafter E3 and E4) were the paint materials. The CSNP Aerosil A380 


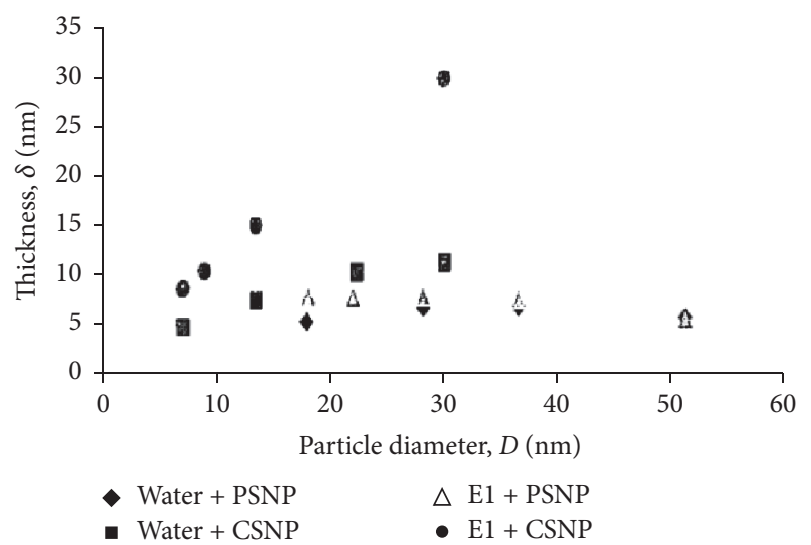

(a)

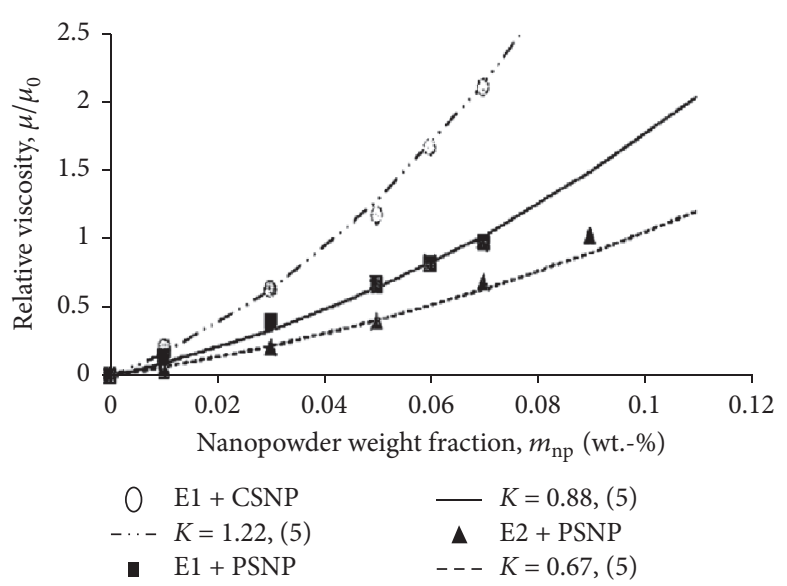

(b)

Figure 4: Comparative relations: (a) "particle size, thickness of the particle associated layer," and (b) "concentration of the nanopowder, viscosity of a liquid composition," due to different diffusion ability of the CSNP and PSNP.

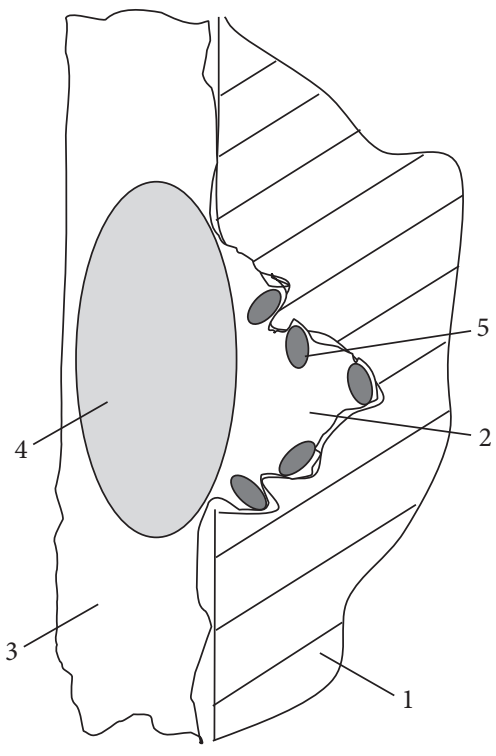

(a)

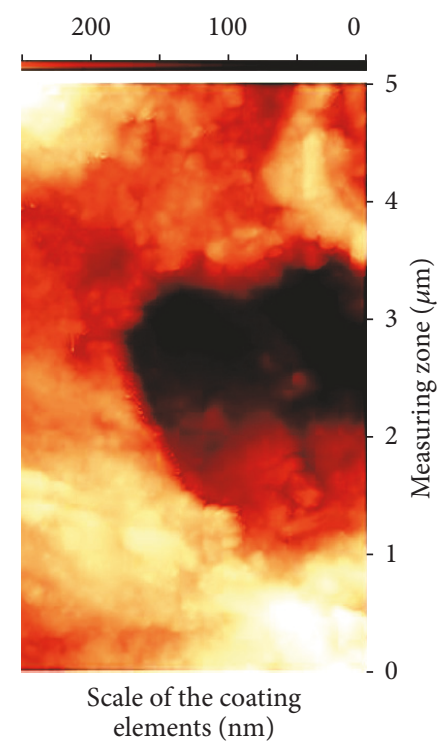

(b)

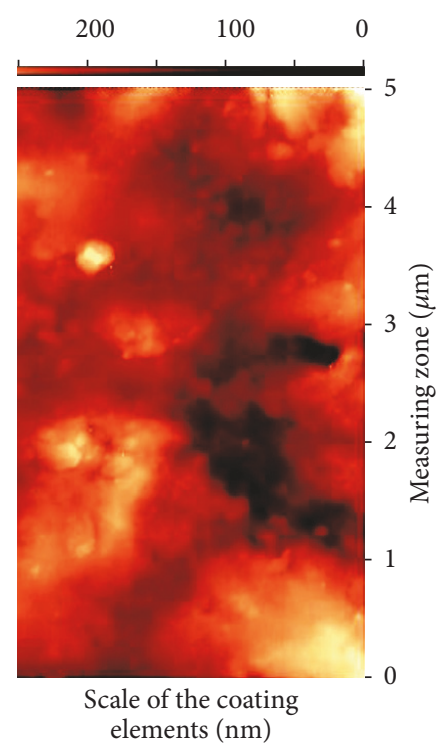

(c)

FIGURE 5: Synthesis of redundant structural constraints and chemical bonds in a PCS by using the PSNP: (a) a model of the constraints, where 1-5 are the painted surface, microcrack, coating layer, paint microparticle, and nanoparticle agglomerates of the modifiers; (b)-(c) AFM of the bonds before and after the modification.

$\left(S_{\mathrm{CSNP}}=380 \mathrm{~m}^{2} / \mathrm{g}\right)$ and PSNPs Tarkosil T50 $\left(S_{\mathrm{PSNP}}=50 \mathrm{~m}^{2} / \mathrm{g}\right)$ and T80 $\left(S_{\text {PSNP }}=80 \mathrm{~m}^{2} / \mathrm{g}\right)$ were selected as the modifiers on the factory testing, as the best results shown through the modeling studies.

3.2.1. Paint Material Savings. The WFT of a coating was spot-checked and measured during the paint application. The reference PCSs of the LBH $250 \times 105 \times 5 \mathrm{~mm}$ each and 30-50 replicate specimens per paint material type were used. The DFT of each primer and paint coating was measured 7 days later using electromagnetic gauges. Statistical analysis [43] of the DFT data was carried out for comparison of two efficiency indexes: a paint material saving and loss of the DFT (see examples of the data processing in Figure 6). In the graphs, each "1" shows the index for a composition TC + PSNP1, E3 + PSNP1, or E4 + PSNP1; each " 2 " is the index for TC + PSNP2, $\mathrm{E} 3+\mathrm{PSNP} 2$, or E4 + PSNP2; and " 3 " indicates the indexes for TC + CSNP, E3 + CSNP, or E4 + CSNP. The weight fractions of CSNP and PSNP are identical in a composition per paint material type. Finally, index " 4 " is related to the efficiency when using TC, E3, or E4 without the modifiers.

As seen in Figure 6, the modifiers essentially changed the indexes. However, the indexes of the paint materials premixed with PSNP are higher and more stable than with application of the CSNP. A source of ambiguity in using the CSNP or PSNP could be the following. The associated layers of the 


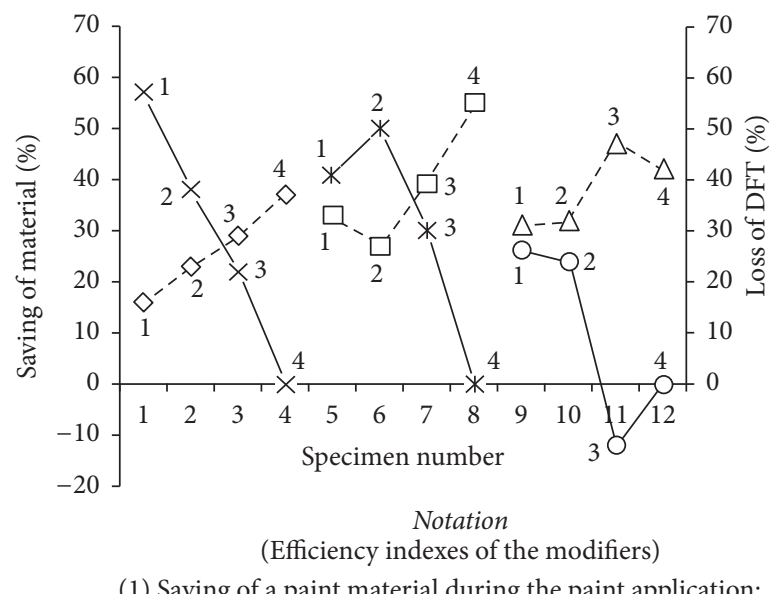

(1) Saving of a paint material during the paint application:

$$
\times \text { for TC } * \text { for E3 } O \text { for E4 }
$$

(2) Loss of the coating thickness after drying:

$$
\diamond \text { for TC } \square \text { for E3 } \quad \triangle \text { for E4 }
$$

FIGURE 6: Assessment of a comparative consumption of the paint materials with and without use of the modifiers.

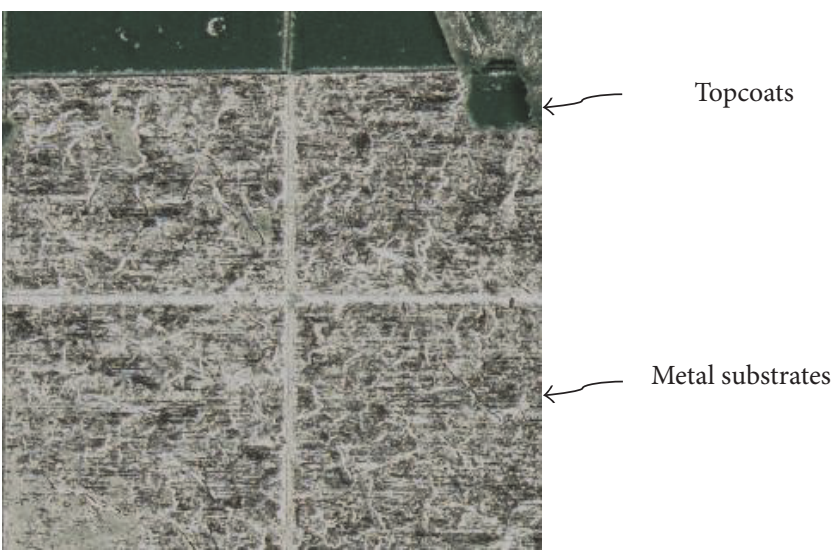

(a)

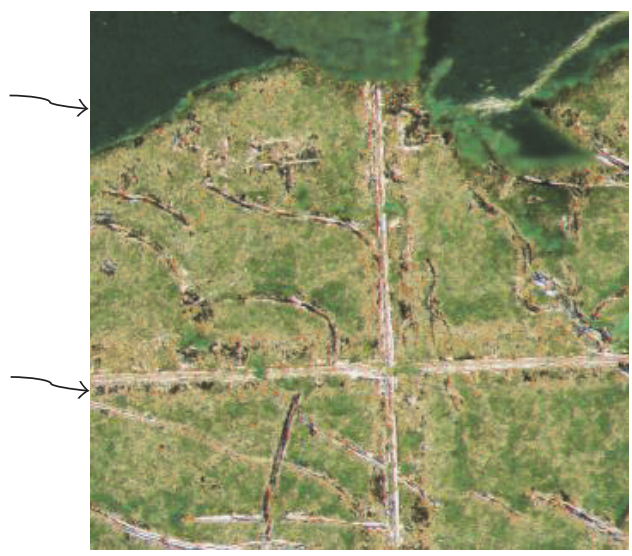

(b)

Figure 7: Micrographic test. Comparative adhesion of the PCSs when using (a) a polyurethane resin based paint with the CNPS and (b) the same paint with the PNPS. The cross-cut mesh is $0.7 \times 0.7 \mathrm{~mm}$.

PSNP particles are more active. Therefore, stronger chemical bonds are synthesized between the paint molecular chains and the PSNP particles and their agglomerates. This increased the strength of the DFT. At the same time, much of the solid residual remains in the coating when using the PSNP. This resulted in a DFT with a smaller loss and finally a greater paint material saving.

\subsubsection{Control of Adhesion and Cohesion Strength to Improve} a PCS. The cross-cut and pull-off methods were used to estimate and compare the PCSs' adhesion. Statistical analysis was done in correspondence with standard classifications. Initially, the adhesion by the cross-cut method was considered acceptable in comparison with the standard. All modified PCSs were classified as $4 \mathrm{~B}$ and $5 \mathrm{~B}$. At the same time, the test by the micrographic technology [39] showed that the adhesion of PCSs modified with PSNP was much higher than of PCSs modified with CSNP. For instance, Figure 7(a) demonstrates that the spots of the paint (green color) mixed with the CSNP are very small and cover a minor part of the painted surface. Meantime, the spots of the same paint mixed with the PSNP cover the painted surface almost completely (see Figure 7(b)).

The micrographic technology makes possible comparison of the cohesion between the PCS elements as well. Figure 8 shows interfacial shears of the primer and topcoat. The spots of the primer mixed with the CSNP are spacious (Figure 8(a)). The spots of the primer mixed with the PSNP cover a minority of painted surface (Figure 8(b)).

Statistical analysis of the pull-off test data (in addition to the cross-cut test) showed that the adhesion of a PCS modified with PSNP was higher than the upper range limit of the standard [38] and the adhesion of an unmodified PCS. 


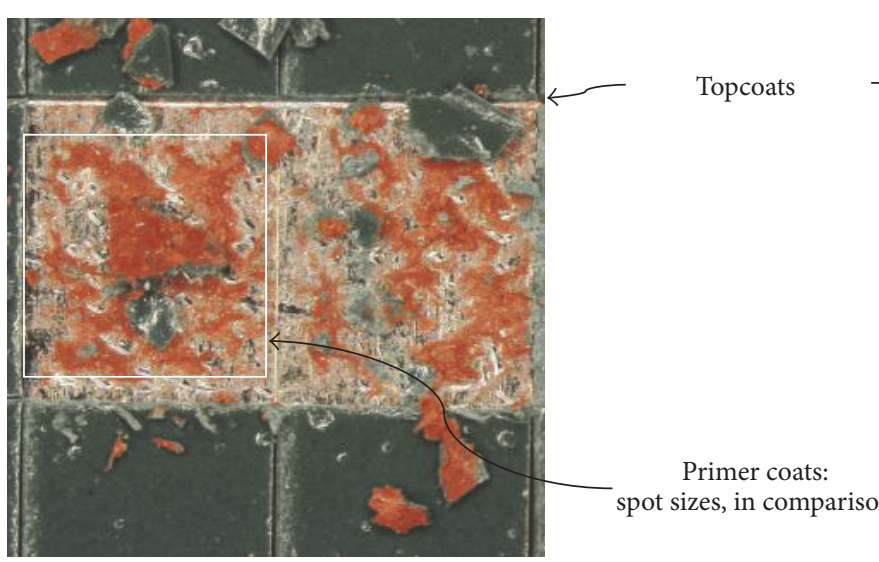

(a)

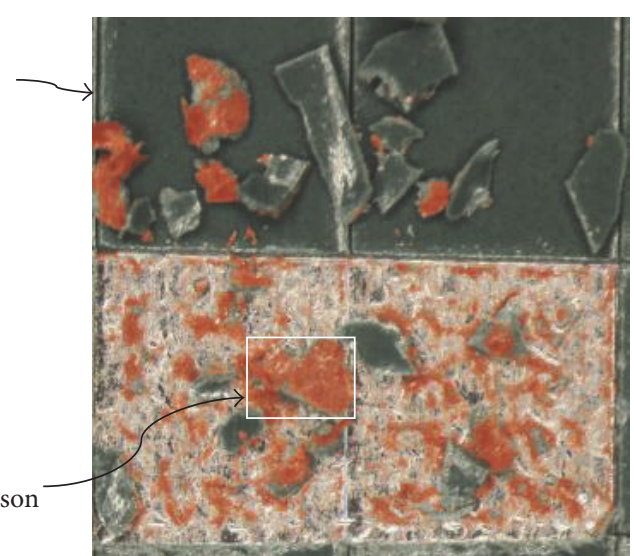

(b)

FIgURE 8: Micrographic test. Comparative cohesion of the PCSs when using (a) the primer and finish paint (topcoat) with the CNPS and (b) the same materials with the PNPS. The cross-cut mesh is $0.7 \times 0.7 \mathrm{~mm}$.

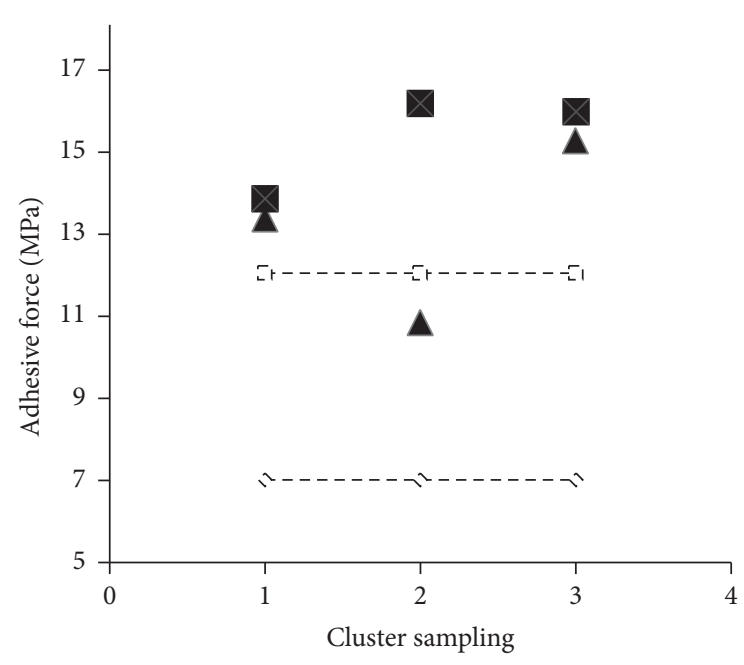

Notation

- - The limits of adhesive force by the standard [38]

$\checkmark$ RMS of the maxima of the force measured

A RMS of the minima of the force measured

FIGURE 9: Adhesion control of an unmodified PCS (cluster 1) and the PCSs modified with CSNP (2) or PSNP (3).

However, the adhesion of a PCS modified with CSNP had an essential parameter spread (Figure 9).

3.2.3. Corrosion Resistance to Salt Water. The specimens of the PCSs modified with CSNP and PSNP were dipped into $\mathrm{NaCl}$ solution for more than 240 hours. After moisture removal from the painted surfaces, the cross-cut test of the coatings was carried out. The cross-cut mesh had an intensity of $2 \times 2 \mathrm{~mm}$ (see samples in Figure 10). Adhesion of the PCSs was classified as $4 \mathrm{~B}$. Besides, the PCSs modified with PSNP showed excellent appearance.

Then, the corrosion spread was statistically analyzed. The density of air-cells and air-water bubbles that arisen under
TABLE 1: Comparative corrosion resistance of modified PCSs to salt water.

\begin{tabular}{lcc}
\hline \multirow{2}{*}{ Controlled variable of a PCS } & \multicolumn{2}{c}{ Type of the modifier } \\
& CSNP & PSNP \\
\hline Density of air-cells and bubbles $(\%)$ & $<15$ & 0 \\
Breadth of corrosion spread (mm) & $<0.5$ & 0 \\
\hline
\end{tabular}

the paint coating and also the breadth of the corrosion spread were evaluated by using the formulae [40]

$$
\begin{aligned}
& D=\frac{A_{d}}{A_{p}} \cdot 100 \%, \\
& H=\frac{\left(H_{c}-H_{0}\right)}{2},
\end{aligned}
$$

where $A_{d}$ is the surface area with the air-cells and bubbles $\left(\mathrm{mm}^{2}\right)$ and $A_{p}$ is the surface area of the coating $\left(\mathrm{mm}^{2}\right)$. Any such defects that occurred at a distance of $10 \mathrm{~mm}$ from the edge of the surface painted were not taken into account. Here $H=\left(\Sigma h_{i \max }\right) \cdot n^{-1}$ is the average breadth of corrosion spread $(\mathrm{mm}), h_{i \max }$ is the maximum of the breath within the line of each $10 \mathrm{~mm}$ cut, and $n$ is the number of cuts; $H_{0}=0.5 \mathrm{~mm}$ is the initial breadth of the cut.

In spite of good adhesion initially, one can predict that the PCSs modified with the CSNP will rapidly deteriorate in a continuous operation due to air-cells randomly distributed under coating (see Figure 10(a) and statistical data in Table 1).

\section{Operation of Modified Coatings in Harsh Environmental Conditions}

Modification of the paint coatings through the dosing, mixing, and vibration-and-pulse dispersion of certain (small) volumes of different PSNPs into a paint or/and primer has been used to improve the PCSs operating in harsh environment. These are the outside surfaces of the structures 


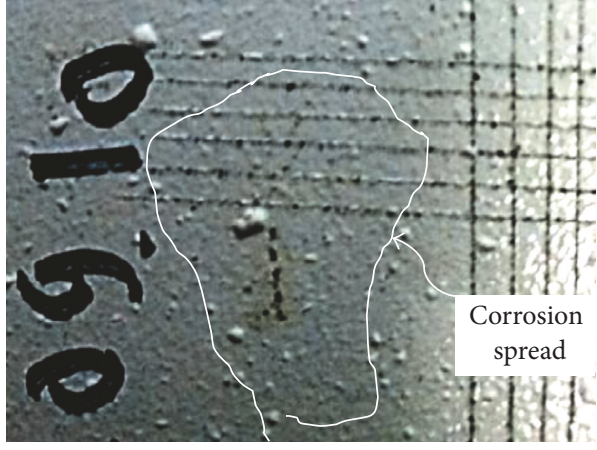

(a)

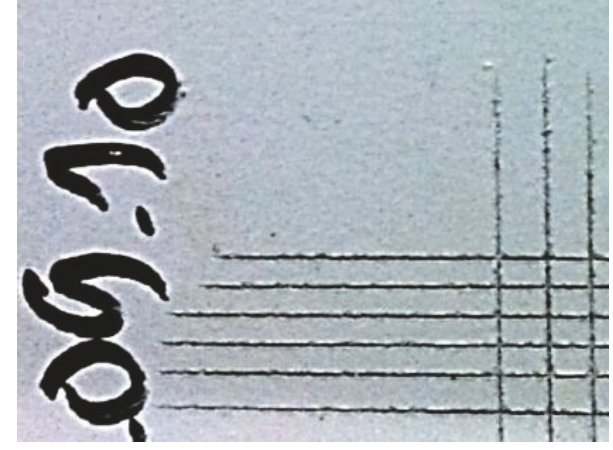

(b)

FIgURE 10: Corrosion resistance of a modified PCS to salt water when using (a) the CSNP and (b) the PSNP.

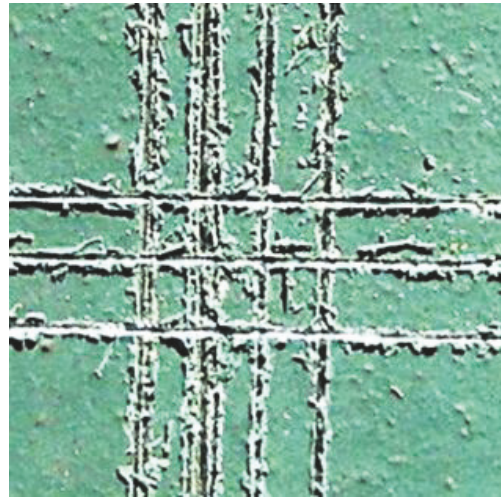

(a)

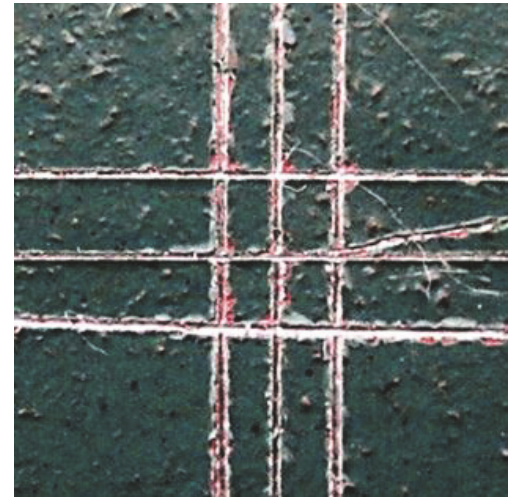

(b)

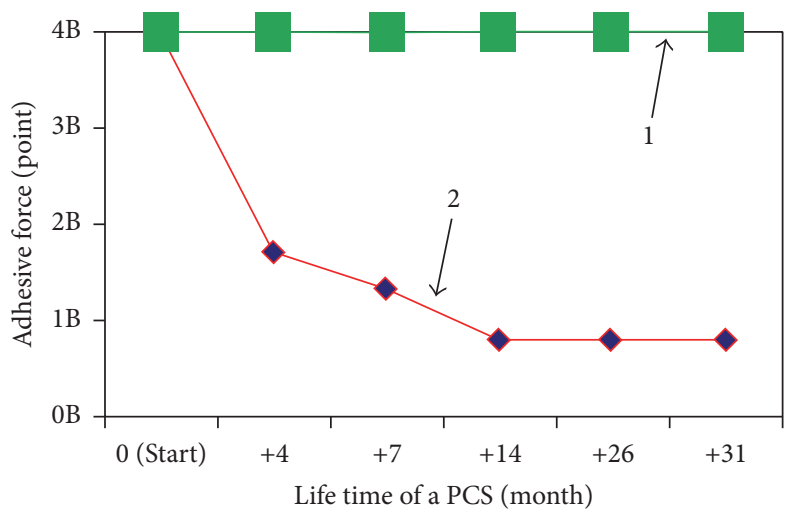

(c)

FIGURE 11: Adhesion and appearance (a)-(b) of an unmodified external PCS and PCS modified with the PSNP, after 31 months of operation in the same environmental conditions, where the cross-cut mesh is $2 \times 2 \mathrm{~mm}$ and (c) comparative degradation of modified (1) and unmodified (2) PCSs.

of railroad rolling stock (carbodies) and infrastructure (housings of the automatic control systems), civil and industrial engineering (metal supporting beams and roofing), automobiles (bodies and bumpers), helicopters (fuselages and blades), and others [14, 34, 44].

Figure 11 demonstrates a comparative level of degradation of a multicoat PCS made of the off-the-shelf finish paint and primer and a PCS made of the same paint materials modified with the PSNP $\left(S_{\text {PSNP }}=100 \mathrm{~m}^{2} / \mathrm{g}\right)$. These PCSs are the opposite sides of a railroad carbody painted within a planned overhaul. Then, this testing car operated at a daily long-distance passenger train within three years. The train operated in harsh environmental conditions. For instance, a regular one-way train trip from the city in subarctic 
climate to the city in humid subtropics has taken four days. And in a winter the ambient air temperature changed from $-37 \cdots-47^{\circ} \mathrm{C}$ to $+21 \cdots+29^{\circ} \mathrm{C}$ within the trip.

As seen in Figures 11(a) and 11(c) (graph 2), unmodified PCS degraded earlier than predicted by the standard and much earlier than PCS modified with the PSNP. However, the modified PCSs kept safe initial levels of the adhesion and appearance (see Figure 11(b) and graph 1 in Figure 11(c)). These experimental data could also be taken into account to compare the efficiency of the PSNPs and CSNPs, because such CSNPs are used in the production of the finish paints and primers as a thixotropic agent mentioned above in Section 2.1.

\section{Conclusions}

Silica nanopowders with analogous or similar general properties synthesized by chemical and physical methods have essential differences in the particle formation and adsorption mechanisms. This leads to higher diffusion capacity of particle surfaces of the "physical" nanopowders and can give important advantages that are reasonable in terms of their usefulness and cost in the paint application and operation of modified protective multicoat systems. In many practical cases, rational choice of silica nanopowders can allow reducing the consumption of paint materials by $30 \%$ or more, at least 2-3 times increasing of the coating adhesion and hence the system life. This was proved through comparative studies and a long-time practical use of modified protective coating systems for railroad rolling stock and outdoor automatic control systems, deck superstructures, helicopters, land vehicles, and other types of machines and infrastructure facilities operating in harsh and extreme environment.

\section{Conflicts of Interest}

The authors declare that there are no conflicts of interest regarding the publication of this paper.

\section{Acknowledgments}

This work was supported by the Russian Ministry of Education and Science by Grant 16.1930.2014/K and by a 2015-2016 Research Fund of the University Of Ulsan, Korea. The authors thank colleagues from Siberian Division of Russian Academy of Science and Novosibirsk State Technical University for help in preparing the test samples and fruitful discussions. Special thanks are due to the Coating \& Combination Engineering Co., Ltd. (Ulsan, Korea), for the technical support in the factory testing.

\section{References}

[1] Global nanoparticle analysis market research report 2016, 9Dimen Research, June 2016, http://www.reportlinker.com.

[2] “The global market for zinc oxide nanopowders," Strategic Report, Future Market, 2015.

[3] B.-F. Zhang, Y.-M. Shen, and M.-H. Du, "Study on preparation and properties of $\mathrm{Fe}_{2} \mathrm{O}_{3}$ nanopowders," in Proceedings of the
International Conference on Advances in Energy and Environmental Science (ICAEES '15), pp. 1-5, July 2015.

[4] X. Chen and S. S. Mao, "Titanium dioxide nanomaterials: synthesis, properties, modifications and applications," Chemical Reviews, vol. 107, no. 7, pp. 2891-2959, 2007.

[5] R. K. Iler, The Chemistry of Silica: Solubility, Polymerization, Composition and Surface Properties and Biochemistry of Silica, Wiley-Interscience Publication, New York, NY, USA, 1979.

[6] A. P. Legrand, Ed., The Surface Properties of Silicas, John Wiley \& Sons, New York, NY, USA, 1998.

[7] L. Canham, Ed., Handbook of Porous Silicon, Springer, Berlin, Germany, 2014.

[8] G. Leder, T. Ladwig, V. Valter, S. Frahn, and J. Meyer, "New effects of fumed silica in modern coatings," Progress in Organic Coatings, vol. 45, no. 2-3, pp. 139-144, 2002.

[9] S.-X. Zhou, L.-M. Wu, J. Sun, and W.-D. Shen, "Effect of nanosilica on the properties of polyester-based polyurethane," Journal of Applied Polymer Science, vol. 88, no. 1, pp. 189-193, 2003.

[10] P. K. Namburu, D. P. Kulkarni, A. Dandekar, and D. K. Das, "Experimental investigation of viscosity and specific heat of silicon dioxide nanofluids," Micro and Nano Letters, vol. 2, no. 3, pp. 67-71, 2007.

[11] K. B. Anoop, S. Kabelac, T. Sundararajan, and S. K. Das, "Rheological and flow characteristics of nanofluids: influence of electroviscous effects and particle agglomeration," Journal of Applied Physics, vol. 106, no. 3, Article ID 034909, 2009.

[12] A. Zenerino, T. Darmanin, E. Taffin De Givenchy, S. Amigoni, and F. Guittard, "Connector ability to design superhydrophobic and oleophobic surfaces from conducting polymers," Langmuir, vol. 26, no. 16, pp. 13545-13549, 2010.

[13] S. V. Panin, L. A. Kornienko, S. Piriyaon et al., "Antifrictional composites based on chemically modified UHMWPE. Part 2. The effect of nanofillers on the mechanical and triboengineering properties of chemically modified UHMWPE," Journal of Friction and Wear, vol. 32, no. 4, pp. 269-275, 2011.

[14] B. B. Baldanov, S. P. Bardakhanov, V. N. Goverdovskiy et al., "Surplus clusters. Control of the properties of the multilayered protective-decorative coatings," Nanoindustry, no. 2, pp. 30-33, 2013.

[15] H. M. Said, N. G. N. Salleh, M. S. Alias, and A. W. M. ElNaggar, "Synthesis and characterization of hard materials based on radiation cured bio-polymer and nanoparticles," Journal of Radiation Research and Applied Sciences, vol. 6, no. 2, pp. 71-78, 2013.

[16] F. Jamali, I. Danaee, and D. Zaarei, "Effect of nano-silica on the corrosion behavior of silicate conversion coatings on hotdip galvanized steel," Materials and Corrosion, vol. 66, no. 5, pp. 459-464, 2015.

[17] M. Conradi, G. Intihar, and M. Zorko, "Mechanical and wetting properties of nanosilica/epoxy-coated stainless steel," Materials and Technology, vol. 49, no. 4, pp. 613-618, 2015.

[18] P. Adapala, S. Gaur, R. G. Puri, and A. S. Khanna, “Development and evaluation of nano-silica dispersed polyurethane based coatings for improved anti-graffiti and scratch resistance," Open Journal of Applied Sciences, vol. 5, no. 12, pp. 808-818, 2015.

[19] R. Mueller, L. Mädler, and S. E. Pratsinis, "Nanoparticle synthesis at high production rates by flame spray pyrolysis," Chemical Engineering Science, vol. 58, no. 10, pp. 1969-1976, 2003.

[20] Aerosil ${ }^{\circledR}$-Specialized fumed silica to enhance coatings performance. Evonic Industries, http://www.aerosil.com. 
[21] S. P. Bardakhanov, A. I. Korchagin, N. K. Kuksanov et al., "Nanopowder production based on technology of solid raw substances evaporation by electron beam accelerator," Materials Science and Engineering B, vol. 132, no. 1-2, pp. 204-208, 2006.

[22] A. V. Nomoev, S. P. Bardakhanov, V. V. Syzrantsev, and V. T. Lygdenov, "Determination of thermal conductivity of silica dioxide Tarkosil T-50 nanopowder by laser flash technique," Journal of Engineering Thermophysics, vol. 25, no. 2, pp. 174-181, 2016.

[23] Marine protective coatings, http://www.kccworld.co.kr.

[24] DER 300-Epoxy resin, http://www.westernamspec.com.

[25] Chemical products-Epoxy, http://www.sverdlova.ru.

[26] Crystallography open database, http://www.crystallography .net.

[27] Y. A. Abzaev, N. O. Kopanitsa, V. A. Klimenov et al., "Structural state modeling of amorphous Tarkosil ${ }^{\circledR}$," Transactions of Tomsk State University of Architecture and Civil Engineering, no. 3, pp. 121-133, 2015.

[28] L. N. Mazalov, V. A. Varnek, S. P. Bardakhanov et al., "Analysis of amorphous silicas properties," Research Report 594, Novosibirsk State University, Novosibirsk, Russia, 1994.

[29] A. I. Bulavchenko and P. S. Popovetsky, "Electrokinetic potential of nanoparticles in reverse AOT micelles: photometric determination and role in the processes of heterocoagulation, separation, and concentration," Langmuir, vol. 26, no. 2, pp. 736-742, 2010.

[30] A. N. Nesterov, A. M. Reshetnikov, A. Y. Manakov et al., "Promotion and inhibition of gas hydrate formation by oxide powders," Journal of Molecular Liquids, vol. 204, pp. 118-125, 2015.

[31] V. V. Syzrantsev, A. P. Zavyalov, and S. P. Bardakhanov, "The role of associated liquid layer at nanoparticles and its influence on nanofluids viscosity," International Journal of Heat and Mass Transfer, vol. 72, pp. 501-506, 2014.

[32] S. P. Bardakhanov, I. V. Vasiljeva, N. K. Kuksanov, and S. V. Mjakin, "Surface functionality features of nanosized silica obtained by electron beam evaporation at ambient pressure," Advances in Materials Science and Engineering, vol. 2010, Article ID 241695, 5 pages, 2010.

[33] A. I. Korchagin, S. N. Fadeev, S. P. Bardakhanov et al., "A method and devise for obtaining a superdispersed silicon dioxide, and the silica nanopowder," RU Patent 2067077, 1996.

[34] V. T. Lygdenov, S. P. Bardakhanov, V. N. Goverdovskiy et al., "A method of paint coating based on chlorinated polyvinyl chloride and glyphtal resin,” RU Patent 2465293, 2012.

[35] ISO, "Paints and varnishes-corrosion protection of steel structures by protective paint systems-measurement and acceptance criteria for the thickness of dry films on rough surfaces," ISO 19840, 2012.

[36] ASTM, "Standard practices for measurement of dry film thickness of protective coating systems by destructive means," ASTM D4138, 2013.

[37] ASTM, "Standard test method for evaluating adhesion by knife," ASTM 6677, 2012.

[38] ASTM, "Standard test method for pull-off strength of coatings using portable adhesion testers," ASTM D4541, 2009.

[39] V. M. Aniskin, "Microscopic miniatures," http://www.odditycentral.com.

[40] ISO, "Field method for the conductometric determination of water-soluble salts," ISO 8502-9, 1998.
[41] G. K. Batchelor, "The effect of Brownian motion on the bulk stress in a suspension of spherical particles," Journal of Fluid Mechanics, vol. 83, no. 1, pp. 97-117, 1977.

[42] V. V. Syzrantsev, K. V. Zobov, A. P. Zavjalov, and S. P. Bardakhanov, "The associated layer and viscosity of nanoliquids," Doklady Physics, vol. 60, no. 1, pp. 46-48, 2015.

[43] R. C. Sprinthall, Basic Statistical Analysis, Pearson PLC, London, UK, 9th edition, 2011.

[44] S. P. Bardakhanov, V. N. Goverdovskiy, V. T. Lygdenov et al., "Study of effect of nanopowders Tarkosil ${ }^{\circledR}$ on the mechanical properties of enamels," Paints Materials and Their Application, no. 7, p. 32, 2009 (Russian). 

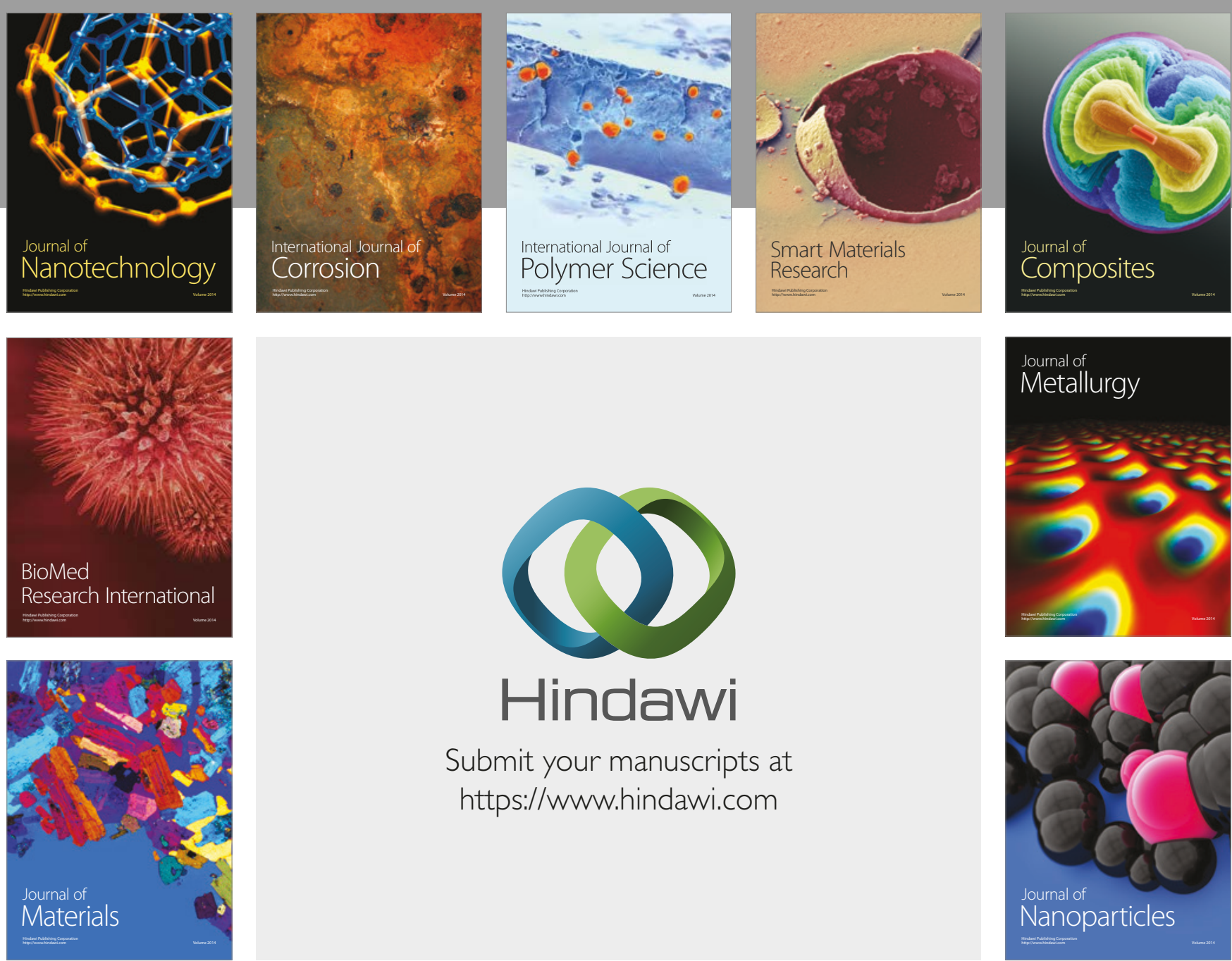

\section{Hindawi}

Submit your manuscripts at

https://www.hindawi.com
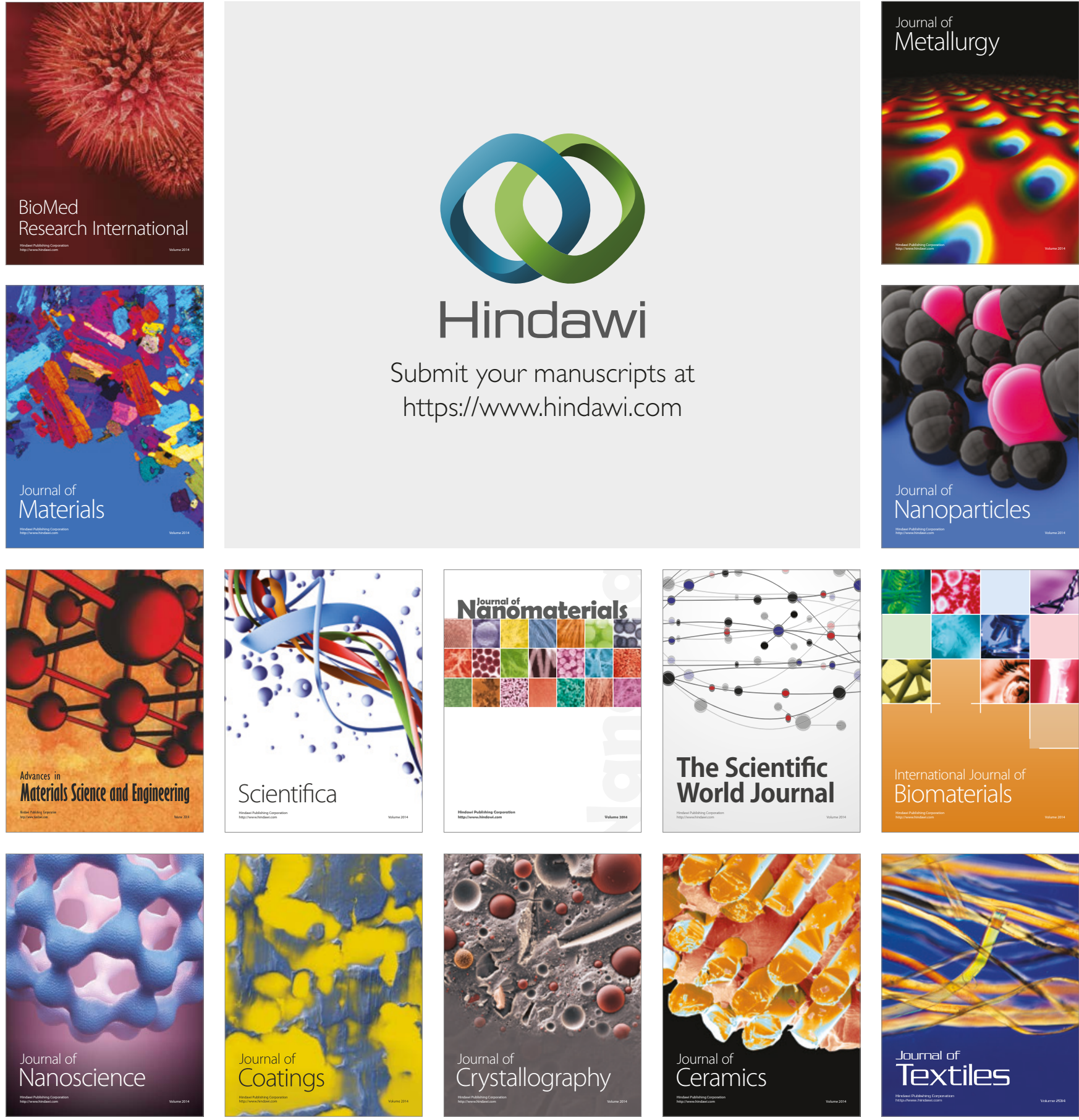

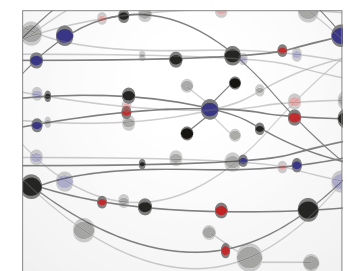

The Scientific World Journal
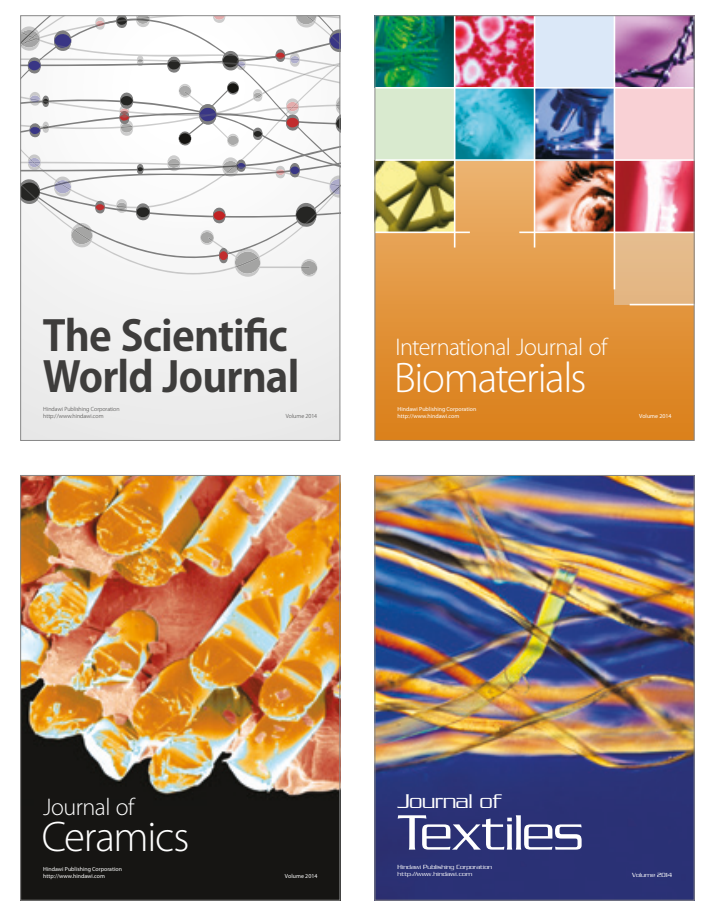